\title{
DOES THE INTERMEDIATE-MASS BLACK HOLE IN LEDA 87300 (RGG 118) FOLLOW THE NEAR-QUADRATIC $M_{\mathrm{bh}}-M_{\text {spheroid }}$ RELATION?
}

\author{
Alister W. Graham ${ }^{1}$, Bogdan C. Ciambur ${ }^{1}$, and Roberto Soria ${ }^{2}$ \\ ${ }^{1}$ Centre for Astrophysics and Supercomputing, Swinburne University of Technology, Victoria 3122, Australia; AGraham@astro.swin.edu.au \\ ${ }^{2}$ International Centre for Radio Astronomy Research, Curtin University, GPO Box U1987, Perth, WA 6845, Australia \\ Received 2015 October 14; accepted 2016 January 15; published 2016 February 17
}

\begin{abstract}
The mass scaling relation between supermassive black holes and their host spheroids has previously been described by a quadratic or steeper relation at low masses $\left(10^{5}<M_{\mathrm{bh}} / M_{\odot} \lesssim 10^{7}\right)$. How this extends into the realm of intermediate-mass black holes $\left(10^{2}<M_{\mathrm{bh}} / M_{\odot}<10^{5}\right)$ is not yet clear, although for the barred Sm galaxy LEDA 87300 , Baldassare et al. recently reported a nominal virial mass of $M_{\mathrm{bh}}=5 \times 10^{4} M_{\odot}$ residing in a "spheroid" of stellar mass equal to $6.3 \times 10^{8} M_{\odot}$. We point out, for the first time, that LEDA 87300 therefore appears to reside on the near-quadratic $M_{\mathrm{bh}}-M_{\mathrm{sph}, *}$ relation. However, Baldassare et al. modeled the bulge and bar as the single spheroidal component of this galaxy. Here we perform a 3-component bulge+bar+disk decomposition and find a bulge luminosity which is 7.7 times fainter than the published "bulge" luminosity. After correcting for dust, we find that $M_{\text {bulge }}=0.9 \times 10^{8} M_{\odot}$ and $M_{\text {bulge }} / M_{\text {disk }}=0.04$-which is now in accord with ratios typically found in Scd-Sm galaxies. We go on to discuss slight revisions to the stellar velocity dispersion $\left(40 \pm 11 \mathrm{~km} \mathrm{~s}^{-1}\right)$ and black hole mass $\left(M_{\mathrm{bh}}=2.9_{-2.3}^{+6.7} \times 10^{4} f_{2.3} M_{\odot}\right)$ and show that LEDA 87300 remains consistent with the $M_{\mathrm{bh}}-\sigma$ relation, and also the near-quadratic $M_{\mathrm{bh}}-M_{\mathrm{sph}, *}$ relation when using the reduced bulge mass. LEDA 87300 therefore offers the first support for the rapid but regulated (near-quadratic) growth of black holes, relative to their host bulge/spheroid, extending into the domain of intermediate-mass black holes.
\end{abstract}

Key words: black hole physics - galaxies: elliptical and lenticular, cD - galaxies: individual (LEDA 087300) galaxies: nuclei - galaxies: photometry - galaxies: structure

\section{INTRODUCTION}

Although stellar mass black holes $\left(<10^{2} M_{\odot}\right.$, e.g., Belczynski et al. 2010) and supermassive black holes $\left(>10^{5} M_{\odot}\right)$ are now known entities (as reviewed in Kormendy \& Ho 2013 and Graham 2015), there remains a paucity of intermediatemass black holes (IMBHs). While there are well over 100 galaxies believed to contain black holes with masses in the range $10^{5}<\left(M_{\mathrm{bh}} f_{3}\right) / M_{\odot}<10^{6}$ (e.g., Greene \& Ho 2004; Jiang et al. 2011; Xiao et al. 2011; Graham \& Scott 2015) ${ }^{3}$, few IMBH candidates are currently known. ${ }^{4}$ However, a battery of indirect methods have been used to imply their existence. For example, perhaps the best such candidate is the hyperluminous X-ray source HLX-1 $\left(M_{\mathrm{bh}}=0.3-30 \times 10^{4} M_{\odot}\right)$, situated offcenter in the lenticular galaxy ESO 243-49 (Farrell et al. 2009, 2014; Soria et al. 2010; Webb et al. 2010, 2012, 2014; Davis et al. 2011; Cseh et al. 2015).

There are three groups of sources touted as IMBH candidates, namely: (i) some highly luminous, off-center, accreting sources in galaxies, such as HLX-1 in ESO 243-49 (these hyperluminous X-ray sources are special cases among

\footnotetext{
3 We use the notation $f_{3}=f / 3$ to denote that a virial factor $f=3$ has been adopted. If the actual virial factor $f$ is greater or smaller than 3 , then the black hole masses need to be multiplied by $f / 3$. The notation $f_{4}$ indicates that a virial factor $f=4$ was used to derive the black hole mass.

4 They tend to be referred to as "candidates" because our scientific instruments typically do not have the ability to spatially resolve the Keplerian orbits of the gas and stars under the gravitational dominance of such black holes in other galaxies. There also remains uncertainty as to the nature and mass of many of the ultraluminous X-ray sources which might (not) be IMBHs (e.g., Feng \& Soria 2011; Liu et al. 2013; Ekşi et al. 2015; Fabrika et al. 2015; Lasota et al. 2015; Zhou 2015).

5 Ultraluminous X-ray sources have $L_{X}>3 \times 10^{39} \mathrm{erg}$, while hyperluminous X-ray sources have $L_{X}>10^{41}$ erg (Gao et al. 2003).
}

the so called ultraluminous X-ray sources) ${ }^{5}$; (ii) centrally located black holes in dwarf and late-type spiral galaxies (such as LEDA 87300), and (iii) (quiescent) black hole candidates in massive globular clusters which have been proposed from dynamical modeling (Wyller 1970; Lützgendorf et al. 2013, and references therein) but not yet convincingly detected (e.g., Lanzoni 2015 and references therein). The three groups may or may not have a distinct physical origin. For example, a source that appears as an off-center hyperluminous X-ray source today may have been the nucleus of an accreted and stripped dwarf satellite galaxy (e.g., Drinkwater et al. 2003; Seth et al. 2014), or perhaps an ejected nucleus (e.g., Gualandris \& Merritt 2008; Komossa \& Merritt 2008; Merritt et al. 2009). NGC 2276-3c is one such off-center X-ray source and probable IMBH, with a reported mass of $\sim 5 \times 10^{4} M_{\odot}$ (Mezcua et al. 2015). Another off-center example can be found in the bulgeless disk galaxy NGC 4178, with a reported black hole mass of $0.6 \pm 0.2 \times 10^{4} M_{\odot}$; and the center of this galaxy may harbour another black hole, in the mass range $10^{4}-10^{5} M_{\odot}$ (Secrest et al. 2012). The non-central, ultraluminous X-ray sources in NGC 1313 (X-2, Liu et al. 2012 and Pasham et al. 2014; and X-1, Colbert \& Mushotzky 1999; Miller et al. 2013 and Pasham et al. 2015), and in Zwicky 18 (Kaaret \& Feng 2013) may also signal IMBHs, wih further examples given in Sutton et al. (2012). If such IMBHs (see also Oka et al. 2016) migrate to the centers of bulges through dynamical friction, it stands to reason that they may contribute to the apparent bulge-(black hole) connection if the inward bound IMBH is of significant mass compared to any black hole mass currently at the center of the galaxy in question. Thus, it is thought that the centrally located, intermediate and 
supermassive black holes may not necessarily originate from a single seed, but rather many.

While a few IMBH candidates are known, as noted above, the galaxy LEDA 87300 (referred to as "RGG 118" by Baldassare et al. 2015; hereafter BRGG15) ${ }^{6}$, hosts a particularly interesting IMBH candidate because it is centrally located in this galaxy's bulge. This galaxy can therefore be used to probe the low-mass end of the (black hole mass)-(host bulge) diagrams and scaling relations. These include (i) the $M_{\mathrm{bh}}-\sigma$ relation (Ferrarese \& Merritt 2000; Gebhardt et al. 2000; Park et al. 2012; Kormendy \& Ho 2013; McConnell \& Ma 2013; Sabra et al. 2015; Savorgnan \& Graham 2015) and the offset nature of barred galaxies (Graham 2008; Hu 2008; Graham et al. 2011), (ii) the near-linear $M_{\mathrm{bh}}-M_{\mathrm{sph}}$ relation ${ }^{7}$ (Magorrian et al. 1998; McLure \& Dunlop 2002; Marconi \& Hunt 2003; Häring and Rix 2004; Sani et al. 2011; Beifiori et al. 2012; Vika et al. 2012; Läsker et al. 2014b) and importantly the steeper $M_{\mathrm{bh}}-M_{\mathrm{sph}}$ relation at low masses (Graham \& Scott 2013, 2015; Savorgnan et al. 2016; see also Laor 1998, 2001), and (iii) the $M_{\mathrm{bh}}-n$ relation (Graham et al. 2001; Savorgnan et al. 2013) involving the Sérsic (1963) index of the host spheroid, i.e., the radial distribution of stars.

The nature of these black hole scaling relations at low masses is not only of interest for probing into the realm of the largely "missing" population of IMBHs, but is expected to provide insight into the seed black holes that grew into supermassive black holes (e.g., Madau \& Rees 2001; Miller \& Hamilton 2002; Portegies Zwart \& McMillan 2002; Johnson et al. 2013; Latif et al. 2013). For example, Alexander \& Natarajan (2014), Madau et al. (2014) and Lupi et al. (2016) illustrate how stellar mass black holes can rapidly grow into $10^{4} M_{\odot}$ black holes via radiatively inefficient super-critical accretion, bypassing studies which start with initial seed black holes with masses $\sim 10^{5} M_{\odot}$. While Kormendy \& Ho (2013) advocate that black holes in low-mass (pseudo)bulges are randomly offset to the lower right of the $M_{\mathrm{bh}}-M_{\mathrm{sph}}$ relations defined by high-mass "classical" bulges — an idea introduced by Graham (2008) and $\mathrm{Hu}(2008)$ _Graham (2012, 2015) has since shown that a steeper than linear relation exists for pseudobulges and low-mass classical bulges alike. In particular, a near-quadratic relation was found for the Sérsic spheroids $\left(M_{\mathrm{bh}} \propto M_{\mathrm{sph}, *}^{2.22 \pm 0.58}\right)$ while a near-linear relation was found for the core-Sérsic spheroids $\left(M_{\mathrm{bh}} \propto M_{\mathrm{sph}, *}^{0.97 \pm 0.14}\right)$. Savorgnan et al. (2016) have since revealed that an alternative division may be between the early-type (red sequence) galaxies (with $M_{\mathrm{bh}} \propto M_{\mathrm{sph}, *}^{1.04 \pm 0.10}$ ) and the late-type (blue sequence) galaxies which define a relation $M_{\mathrm{bh}} \propto M_{\mathrm{sph}, *}^{2}-M_{\mathrm{sph}, *}^{3}$. Using virial black hole masses, the bend in the $M_{\mathrm{bh}}-M_{\mathrm{sph}}$ relation can now be seen all the way down to $M_{\mathrm{bh}}=10^{5} M_{\odot}($ Graham \& Scott 2015). Spheroids with IMBH candidates are needed to test if this steep relation continues to yet lower masses.

BRGG15 did not explore whether the $5 \times 10^{4} f_{4} M_{\odot}$ black hole in LEDA 87300 agrees (or not) with the steep $M_{\mathrm{bh}}-M_{\mathrm{sph}}$,* relation. Figure 1 shows the location of LEDA 87300 in the $M_{\mathrm{bh}}-M_{\mathrm{sph}, *}$ diagram according to the masses derived by BRGG15 for LEDA 87300 . Importantly, it additionally shows, for the first time, where this IMBH resides relative to

\footnotetext{
6 These authors renamed LEDA 87300 after the initials of their previous paper which had included this galaxy in their sample: "Reines, Greene, and Geha".

7 The host spheroid's stellar mass, $M_{\mathrm{sph}, *}$, and dynamical mass, $M_{\mathrm{sph}, \mathrm{dyn}}$, have been used in different studies.
}

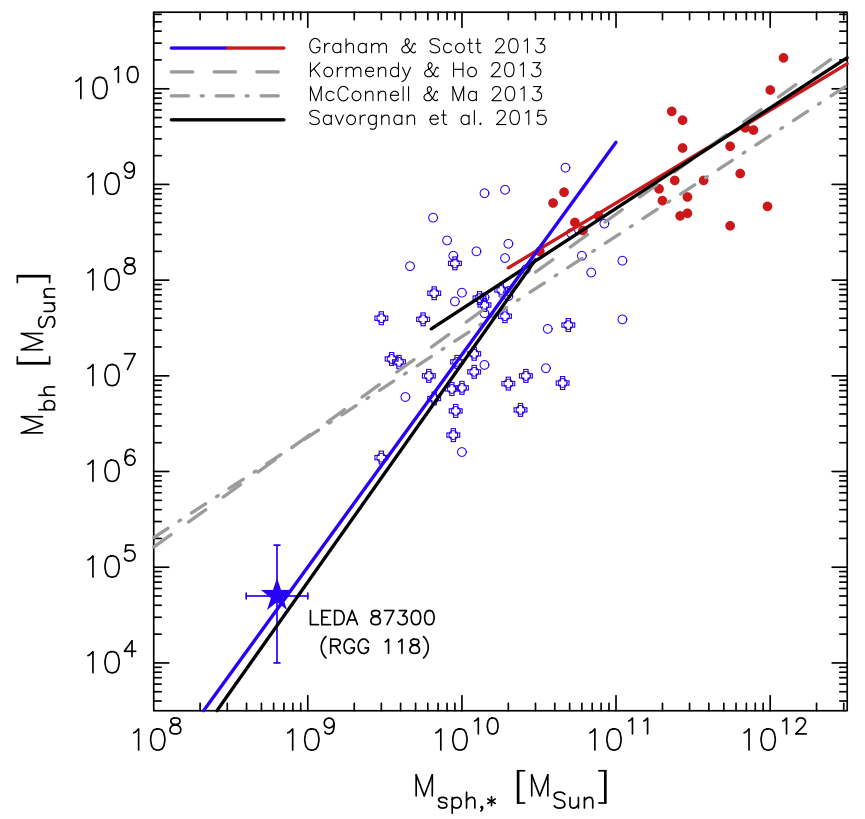

Figure 1. Location of LEDA 87300 in the $M_{\mathrm{bh}}-M_{\mathrm{sph}, *}$ diagram according to Baldassare et al. (2015). The near-quadratic relation from Scott et al. (2013) for Sérsic galaxies (blue points) is shown in blue, and for core-Sérsic galaxies (red points) in red. Barred galaxies are denoted by the crosses. The near-quadratic relation from Savorgnan et al. (2015) for spiral galaxies is shown in black (derived using the modified FITEXY routine from Tremaine et al. 2002 in a symmetrical manner) along with Savorgnan et al.'s near-linear relation for early-type galaxies.

the extrapolation to low masses of the near-quadratic scaling relation constructed at higher masses. The agreement is striking. This is important because, if confirmed, it suggests that we can predict the masses of (at least the more massive) IMBHs.

It is obviously important to perform a correct decomposition of a galaxy's light if we are to obtain the physical properties of the spheroidal component and properly explore the (black hole)-(host spheroid) connection. This is true at both ends of the mass spectrum. For example, isolating the spheroid light from that of the galaxy has recently resulted in the discovery of the missing population of compact, massive spheroids observed at high redshifts (referred to as red nuggets by Damjanov et al. 2009) but previously thought to be absent in the nearby universe (Graham et al. 2015). At the same time, other studies which fit large-scale disks to some of these nearby galaxies which actually contain intermediate-scale disks has resulted in the under-estimation of the spheroid mass and thus the erroneous claims of overly high $M_{\mathrm{bh}} / M_{\mathrm{sph}, *}$ ratios, as explained in Savorgnan \& Graham (2016a).

Here we provide an independent decomposition for the barred galaxy LEDA 87300, to check if its low-mass spheroidal component follows the near-quadratic $M_{\mathrm{bh}}-M_{\mathrm{sph}}$,* relation as seen in Figure 1. Although BRGG15 performed a bulge/disk decomposition, they reported a bulge-to-disk ratio of $\sim 0.3$. This is unusually high for an Sm galaxy (see Graham \& Worley 2008), and appears to have arisen because they lumped the bar plus bulge light together, fitting for what we call the "barge." This also means that the Sérsic index for the bulge component of this galaxy is yet to be measured.

In Section 2 we present the imaging data and our decomposition analysis, refitting for both the "barge" + disk and the bar+bulge+disk. To be thorough, in Section 3 we 
additionally revisit the derived black hole mass, the spheroid velocity dispersion, and the X-ray data which suggested the presence of an active galactic nucleus (AGN). Specifically, in Section 3.1 we revise the nominal black hole mass using the optimal virial $f$-factor for barred galaxies. In Section 3.2 we provide an estimate of the stellar rather than the gas velocity dispersion, and in Section 3.3 we report on our confirmation of the X-ray detection made by BRGG15. In Section 4 we present our revised location of LEDA 87300 in the $M_{\mathrm{bh}}-M_{\mathrm{sph}, *}, M_{\mathrm{bh}}-$ $\sigma$, and $M_{\mathrm{bh}}-n$ diagrams. We provide a brief discussion of its location in these diagrams and comment on potential future targets.

BRGG15 did not identify "RGG118" with any pre-existing galaxy, and we therefore provide some additional references and information here. LEDA 87300 is located at R.A. $=1523$ 05.0, decl. $=+114553$ (J2000: Paturel et al. 2000 and is also known as PGC 87300; Paturel et al. 1989). ${ }^{8}$ BRGG15 have reported a virial mass of $5 \times 10^{4} f_{4} M_{\odot}$, with a $(1 \sigma)$ range from $(1-17) \times 10^{4} f_{4} \quad M_{\odot}$, for the black hole located at the center of this cataloged low surface brightness ${ }^{9}$, Sm galaxy (LSBC F725-V01; Schombert et al. 1992). It is also a known HI radio source: AGC 258125 (Haynes et al. 2011) ${ }^{10}$ from the Arecibo Legacy Fast Arecibo L-band Feed Array (ALFALFA) survey. LEDA 87300 has a reported heliocentric velocity of $7278 \mathrm{~km} \mathrm{~s}^{-1}$ in Schombert et al. (1992), which was confirmed by Haynes et al. (2011) who report a value of $7283 \pm 71 \mathrm{~km} \mathrm{~s}^{-1}$. Correcting for (Virgo + Great-Attractor + Shapley)-infall (Mould et al. 2000, via NED) ${ }^{11}$, this heliocentric velocity equates to a recessional velocity of $7936 \pm 77 \mathrm{~km} \mathrm{~s}^{-1}$, or a Hubble expansion redshift of 0.02647. Using $H_{0}=70 \mathrm{~km} \mathrm{~s}^{-1} \mathrm{Mpc}^{-1}, \quad \Omega_{m}=0.3$ and $\Omega_{\Lambda}=0.7$, this gives a (comoving radial) distance of $112.7 \mathrm{Mpc}$ and a physical scale of $532 \mathrm{pc}$ per arcsec (Wright 2006).

\section{OPTICAL IMAGE ANALYSIS}

\subsection{Data}

While there is a 2008 Canada-France-Hawaii Telescope (CFHT)/MegaCam image of the field containing LEDA 87300 (Yee et al. Proposal ID 07AC22), unfortunately LEDA 87300 falls right in the middle of the CCD mosaic gap (which we mention to spare some readers from looking for it there). We have therefore obtained images available from the Sloan Digital Sky Survey (SDSS) Data Release Nine (Ahn et al. 2012). ${ }^{12}$ Figure 2 displays an image of LEDA 087300 created by us using data from two Sloan runs in the DR9 Science Archive ${ }^{13}$, identified by the Run, Camcol, and Field numbers (005322, 2, 0142) and (003996, 6, 0175).

We aligned the $g^{\prime}-, r^{\prime}$-, and $i^{\prime}$-band images from these two runs by rotating and shifting the images, and then we stacked the images from all three filters to increase the signal to noise. It is apparent from Figure 2 that LEDA 87300 is a barred spiral

\footnotetext{
8 The Catalog of Principal Galaxies.

9 "Low surface brightness galaxies" have a central disk surface brightness more than 1 mag $\operatorname{arcsec}^{-2}$ fainter than the canonical Freeman (1970) value of $21.65 \mathrm{~B}$ mag $\operatorname{arcsec}^{-2}$.

${ }^{10}$ The "Arecibo General Catalog" is a private database maintained by Martha P. Haynes and Riccardo Giovanelli.

11 http://ned.ipac.caltech.edu/

12 http://www.sdss3.org/dr9

13 http://dr9.sdss3.org/fields/
}

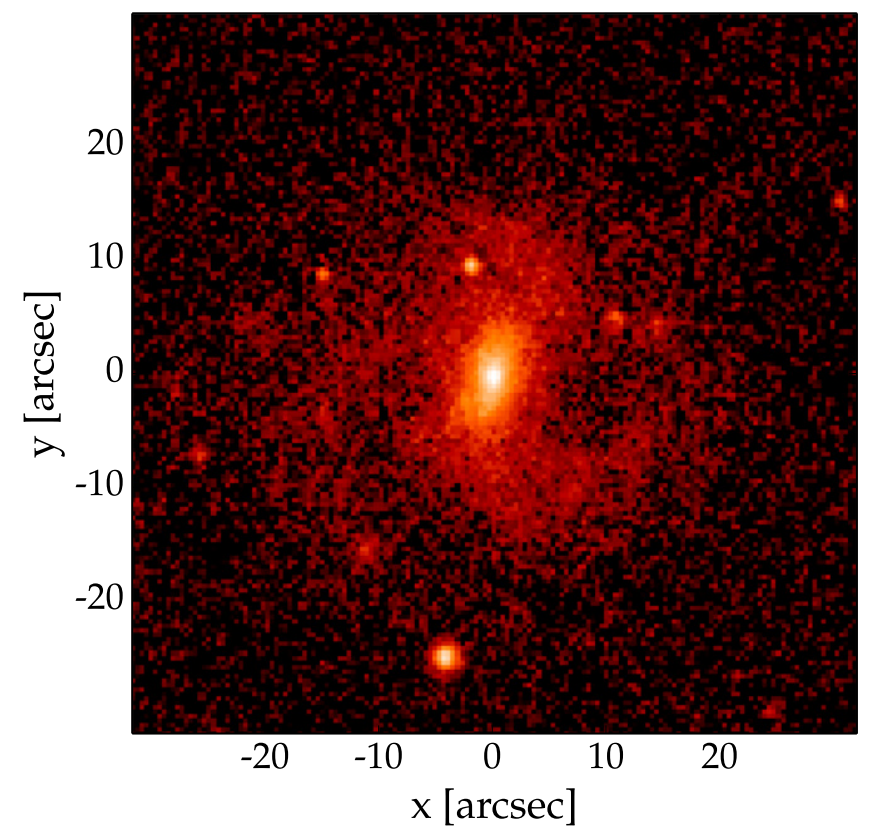

Figure 2. Stacked SDSS $g^{\prime}-, r^{\prime}$-, and $i^{\prime}$-band image of LEDA 87300 (SDSS $\mathrm{J} 152304.96+114553.6$ ) to increase the $\mathrm{S} / \mathrm{N}$ and better reveal the barred spiral galaxy. This image guided the galaxy decompositions subsequently performed on the $g^{\prime}$ - and $r^{\prime}$-images. The scale is such that $10^{\prime \prime}=5.32 \mathrm{kpc}$ in this false color image. East is up and north is to the right.

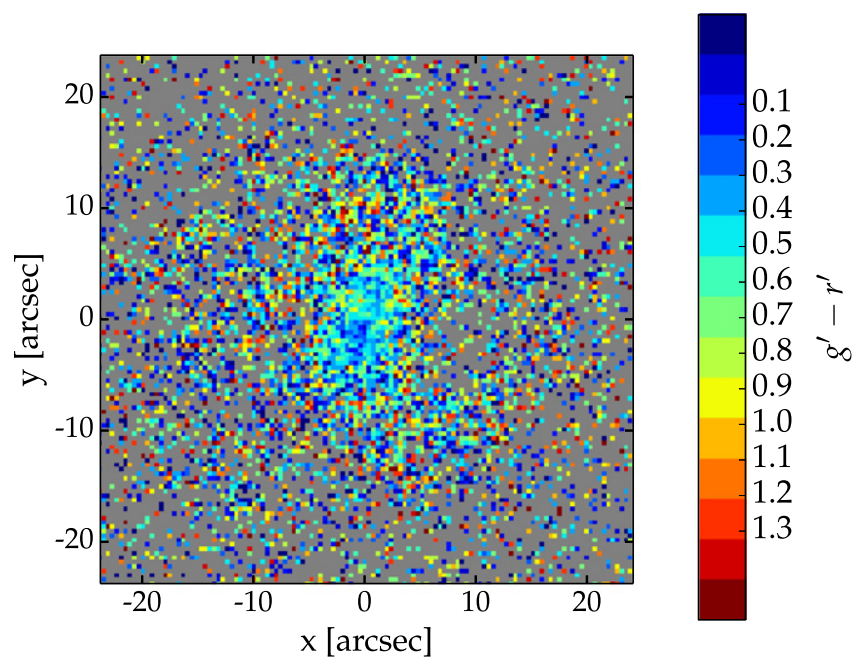

Figure 3. Color map of LEDA 87300 , revealing a relatively red bulge $\left(g^{\prime}-r^{\prime} \sim 0.5\right)$ and blue bar $\left(g^{\prime}-r^{\prime} \sim 0.1-0.2\right)$. The data at larger radii is noisy but has been kept to aid with the recognition of the spiral arms. Correcting for Galactic dust extinction makes the entire image bluer by 0.038. East is up and north is to the right.

galaxy. The morphology of LEDA 87300 is not unusual, and there are plenty of other nearby $(D<30 \mathrm{Mpc})$, relatively faceon, late-type barred spiral galaxies which resemble LEDA 87300, such as NGC 3319, NGC 4519, NGC 7741, and UGC 6983 (Tully 1988). In Figure 3 we show the $g^{\prime}-r^{\prime}$ color map for LEDA 87300. This was created after convolving the images with the better "seeing" with a Gaussian function in order to degrade them to the spatial resolution (1".27) of the poorest image. While the color map is noisy in the outer regions of this pixel-by-pixel representation, without binning, 

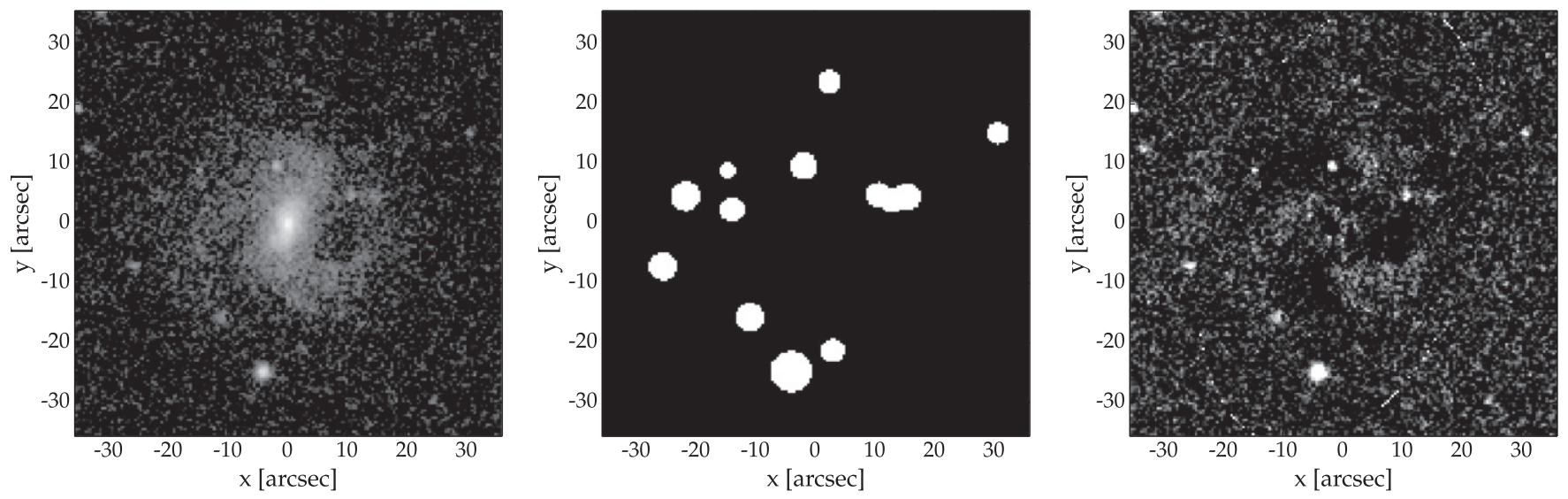

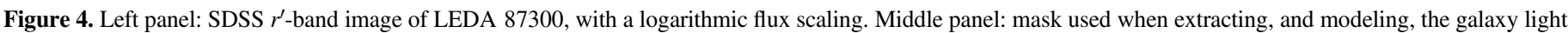

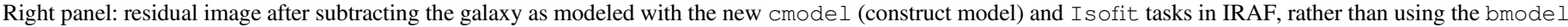

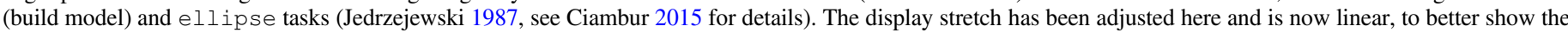
residuals at all radii. A three-armed spiral structure is apparent. The scale is such that $10^{\prime \prime}=5.32 \mathrm{kpc}$,

the inner regions already hint at a somewhat blue bar upon which a small, relatively redder spheroid may be superimposed.

For our analysis, we used the two available data sets to generate a single image for each of the two bands that we modeled. We did this for the $g^{\prime}$ - and $r^{\prime}$-band images, but not the $i^{\prime}$-band image because it is too faint. Galaxy images are not always well approximated by two-dimensional (2D) models whose components have fixed ellipticity $(\epsilon)$ and position angle (P.A.). Instead, we have used one-dimensional (1D) "light profiles" and the associated geometrical profiles which track a galaxy's variation in $\epsilon$, P.A., and isophotal deviation from ellipses-as quantified using Fourier harmonics. Collectively, this suite of one-dimensional profiles describe and recreate the symmetrical elements of a 2D image. The Isofit task (Ciambur 2015) was used to obtain both the major-axis and the geometric-mean-axis (equivalent to a circularized) light profile, and its accompaniments at each radius (i.e., position angle, ellipticity, Fourier terms). As can be seen in the right hand panel of Figure 4, the 1D profiles perform well at representing the galaxy and reveal three spiral arms in the residual map. To avoid redundancy, we do not show the similar set of $g^{\prime}$-band image, mask and residual map, although we do note that the $g^{\prime}$-band profiles could only be reliably extracted to half the radial extent of the $r^{\prime}$-band profile.

\subsection{Modeling}

Having extracted the $g^{\prime}$ - and $r^{\prime}$-band light profiles (shown in Figures 5 and 6), they have then been decomposed into model components (point-source, bar, bulge, disk) using the Profiler software (B.C. Ciambur 2016, in preparation). "Profiler" finds the optimal decomposition of a light profile through an iterative minimization process using the Levenberg-Marquardt algorithm (Marquardt 1963). At each iteration step, the components are summed together and then convolved with the point-spread function (PSF) - using a hybrid (Fast Fourier Transform)-based and numerical convolution scheme - to generate a model profile. Profiler can use a Gaussian, Moffat, or any user-supplied vector to describe the PSF. The best-fit values for the galaxy model parameters are then found by minimizing the root mean square scatter $\Delta_{\mathrm{rms}}=\sqrt{\sum_{i=1, N}\left(\operatorname{data}_{i}-\operatorname{model}_{i}\right)^{2} /(N-\nu)}$, where $N$ is the

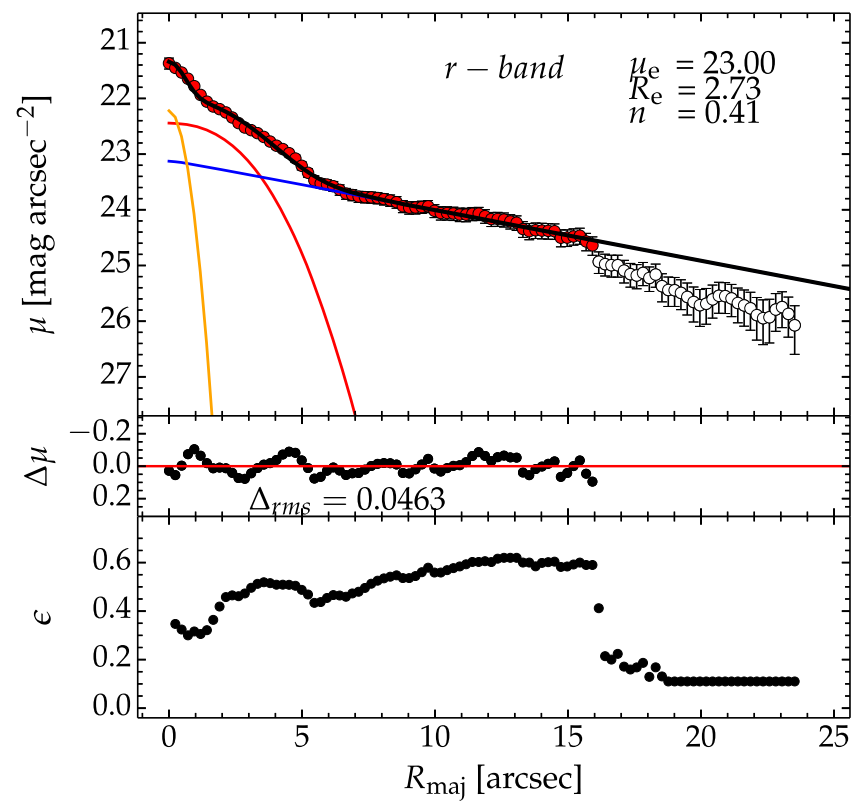

Figure 5. Major-axis $r^{\prime}$-band light profile taken from the left panel of Figure 4 is modeled here with a 3-parameter Sérsic function (red) for the "barge" (bar +bulge), a 2-parameter exponential for the disk (blue), plus a 2-parameter point-source for a necessarily bright AGN (orange). The best-fitting Sérsic parameters are inset in the figure, and the error bars on the data points show our $3 \sigma$ sky-background uncertainty (see Section 2.2.1 for details). Rather than fit a broken exponential model for the disk (e.g., Pohlen \& Trujillo 2006), we truncate the outer data and fit for just the inner exponential. The lower panel shows the ellipticity profile.

number of fitted data points in the light profile and $\nu$ is the number of model parameters.

It should be remembered that $\Delta_{\text {rms }}$ reflects the quality of the global fit. It can therefore be beneficial to also watch the behavior of the residual profile over specific regions of interest. While a (signal-to-noise)-weighted fitting scheme can be used, it is generally preferable not to do so due to the myriad of potential biases (above the Poissonian noise expected from the galaxy) affecting the inner light profiles of galaxies where the signal is highest. Sources of bias can include central dust, a faint AGN, additional components such as a nuclear star cluster or a nuclear disk, and errors in the PSF, all of which become 

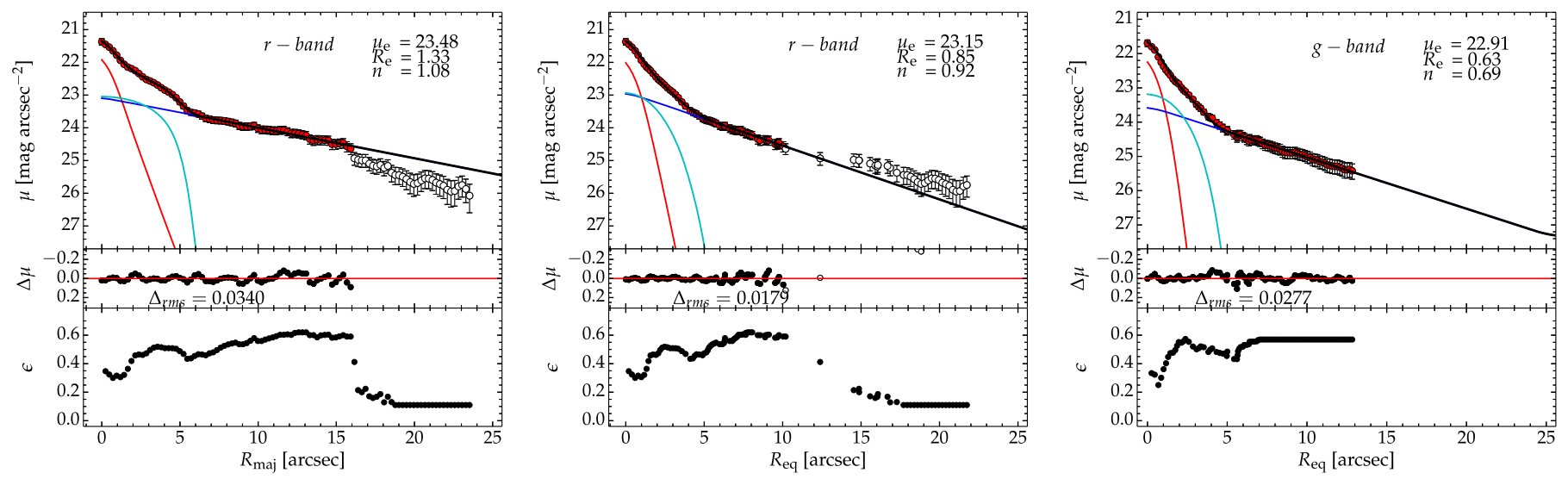

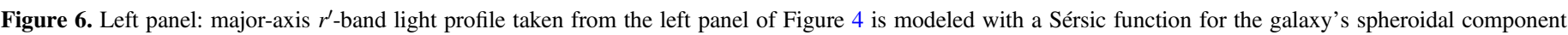

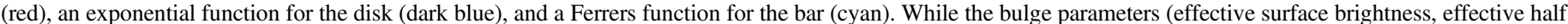

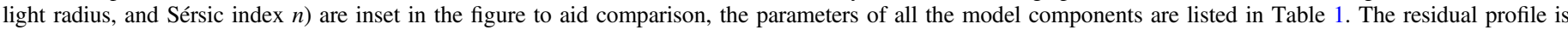

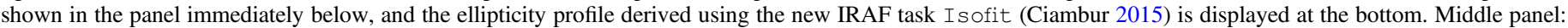

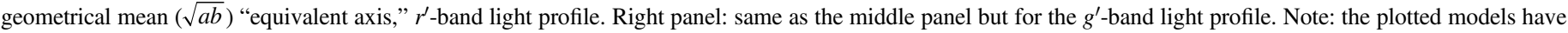

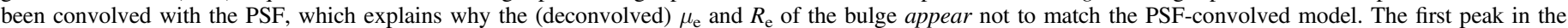

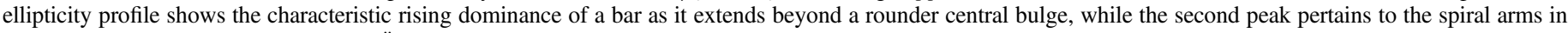
the disk. The radial scale is such that $1^{\prime \prime}=532 \mathrm{pc}$.

more important when using a (signal-to-noise)-weighted scheme. While this danger is considerably reduced through the use $\Delta_{\text {rms }}$, one is more susceptible to biases from an incorrect sky-background subtraction when using this statistic. In the case of LEDA 87300, it has a non-exponential disk which we avoided by restricting the radial range used, and thus we are not using data that could be overly susceptible to errors from the sky-background subtraction process. In passing we note that even if we had modeled the disk with a broken, inner and outer, exponential function (e.g., Pohlen et al. 2002; Pohlen \& Trujillo 2006), it would not have affected our results for the spheroid.

The imexamine task in IRAF was used to fit Gaussian profiles to a dozen stars in each of the final $g^{\prime}$ - and $r^{\prime}$-band images, and the mean FWHM used to characterize our Gaussian PSF. Initially we fit the same model components as BRGG15, namely a point-source, an exponential disk, plus a single Sérsic (1963) $R^{1 / n}$ function $^{14}$ for the combined bar +bulge ("barge"), as shown in Figure 5.

We obtained a notably smaller $r^{\prime}$-band Sérsic index for the barge than BRGG15 (0.41 compared to 1.13), and a barge luminosity which is less than two times as bright. Our uncorrected $r^{\prime}$-band barge magnitude of $19.29 \mathrm{mag}$ and disk magnitude of $16.82 \mathrm{mag}$ give a barge-to-disk flux ratio of $\sim 0.10$. Given that the bulge-to-disk flux ratio is typically just a few percent for Scd-Sm galaxies (e.g., Graham \& Worley 2008), this is additional evidence (beyond simply looking at the $2 \mathrm{D}$ image and the $1 \mathrm{D}$ profile) that the bar is likely contributing to the barge light. ${ }^{15}$ While at some stage bars become bulges within the regime of secular disk evolution (e.g., Hohl 1975; Combes \& Sanders 1981; Combes et al. 1990), and thus one could perhaps argue for the use of barge light rather than bulge light, in the following section we have proceeded by performing a bulge + bar + disk

\footnotetext{
14 A review of the Sérsic function, which was popularized by Caon et al. (1993) and reproduces an exponential and Gaussian profile when $n$ equals 1.0 and 0.5, respectively, can be found in Graham \& Driver (2005).

${ }^{15}$ We obtained a disk $g^{\prime}-r^{\prime}$ color of $0.41 \pm 0.03$ and a bluer barge color of 0.37 , albeit with an uncertainty of 0.05 .
}

decomposition, as done for the galaxies with supermassive black holes (e.g., Läsker et al. 2014a; Savorgnan \& Graham 2016a, and references therein).

\subsubsection{A Bar+Bulge+Disk Decomposition}

We used a modified Ferrers profile to describe the bar (Ferrers 1877; Peng et al. 2010). The best-fitting bulge+bar + disk model parameters are provided in Table 1. Of immediate note is the improved quality of the fit, evinced by the smaller residuals around the inner $\sim 5^{\prime \prime}$ and the reduced rms scatter (Figure 6). With a major-axis, $r^{\prime}$-band half light radius equal to two-thirds of a Kiloparsec, the central component is neither an AGN point source nor a star cluster but instead the bulge component of this galaxy. Its size is typical for late-type spiral galaxies (e.g., Graham \& Worley 2008), further suggesting that there is nothing unusual with LEDA 87300.

BRGG15 included a central point source in their model (see their Figure 3 and our Figure 5), but we do not see evidence of a possible fourth component after our new model has described the three major components of this galaxy. Future Hubble Space Telescope images would be valuable for assessing the contribution/existence of any optical AGN point source. A nuclear star cluster may also be expected, and the prescription in Graham (2016) suggests that it would be a few million solar masses if associated with the black hole in LEDA 87300, and thus it would have an $r^{\prime}$-band magnitude prior to dust extinction of $\approx-24 \mathrm{mag}$ (assuming a stellar mass-to-light ratio of unity). This is too faint for us to detect in the SDSS image. A nuclear disk is yet another option, but again, our attempts to fit a fourth component did not detect anything.

Integrating the surface brightness profiles (to $R=\infty$ ) of the individual model components that were fit to the equivalentaxis light profile (Figure 6) has the advantage that it can be done using spherical symmetry (see the Appendix in Ciambur 2015 to understand how this recovers the total galaxy light). The resultant luminosity of each component is included in Table 1 , and collectively gave a total, uncorrected $g^{\prime}$-band magnitude of $17.17 \mathrm{mag}$ for the galaxy. For reference, Garnier et al. (1996) reported $B_{T}=17.05 \mathrm{mag}$ (RC3 system, de 
Table 1

$r^{\prime}$-band Galaxy Model Parameters

\begin{tabular}{|c|c|c|c|c|c|c|c|c|c|c|}
\hline Component & $\begin{array}{c}\mu_{\mathrm{e}} \text { or } \mu_{0} \\
\left(\operatorname{mag} \operatorname{arcsec}^{-2}\right) \\
(2)\end{array}$ & $\begin{array}{c}R_{\mathrm{e}} \\
\left(\operatorname{arcsec} \mathrm{kpc}^{-1}\right) \\
(3)\end{array}$ & $\begin{array}{c}h_{\text {disk }} \text { or } R_{0, \text { bar }} \\
\left(\operatorname{arcsec~} \mathrm{kpc}^{-1}\right) \\
\text { (4) }\end{array}$ & $\begin{array}{c}(\alpha, \beta)_{\text {bar }} \\
(5)\end{array}$ & $\begin{array}{c}n_{\text {Sersic }} \\
\text { (6) }\end{array}$ & $\begin{array}{c}m \\
(\mathrm{mag}) \\
(7)\end{array}$ & $\begin{array}{c}m^{\text {corr } 1} \\
(\mathrm{mag}) \\
(8)\end{array}$ & $\begin{array}{c}\mathrm{Mag}^{\text {corr } 1} \\
(\mathrm{mag}) \\
(9)\end{array}$ & $\begin{array}{l}m^{\text {corr2 }} \\
(\mathrm{mag}) \\
(10)\end{array}$ & $\begin{array}{c}\mathrm{Mag}^{\mathrm{corr}} \\
(\mathrm{mag}) \\
(11)\end{array}$ \\
\hline \multicolumn{11}{|c|}{ SDSS $r^{\prime}$-band major-axis } \\
\hline Spheroid & 23.48 & $1.33 / 0.71$ & $\ldots$ & $\cdots$ & 1.08 & $\cdots$ & $\cdots$ & $\cdots$ & $\cdots$ & $\cdots$ \\
\hline Disk & 23.06 & $\ldots$ & $11.57 / 6.16$ & $\cdots$ & 1.00 & $\cdots$ & $\cdots$ & $\cdots$ & $\cdots$ & $\cdots$ \\
\hline Bar & 23.05 & $\cdots$ & $5.32 / 2.83$ & $(1.94,0.01)$ & $\cdots$ & $\cdots$ & $\cdots$ & $\cdots$ & $\cdots$ & $\cdots$ \\
\hline \multicolumn{11}{|c|}{ SDSS $r^{\prime}$-band equivalent-axis } \\
\hline Bar & 22.90 & $\cdots$ & $5.82 / 3.10$ & $(9.99,0.01)$ & $\cdots$ & 20.44 & 20.30 & -14.96 & 20.13 & -15.13 \\
\hline Galaxy & $\cdots$ & $\cdots$ & $\ldots$ & $\ldots$ & $\cdots$ & 16.78 & 16.64 & -18.62 & 16.46 & -18.80 \\
\hline \multicolumn{11}{|c|}{ SDSS $g^{\prime}$-band equivalent-axis } \\
\hline Spheroid & 22.91 & $0.63 / 0.34$ & $\ldots$ & $\ldots$ & 0.69 & 21.39 & 21.21 & -14.05 & 20.35 & -14.91 \\
\hline Disk & 23.53 & $\ldots$ & $7.23 / 3.85$ & $\ldots$ & 1.00 & 17.24 & 17.06 & -18.20 & 16.84 & -18.42 \\
\hline Bar & 23.15 & $\ldots$ & $4.61 / 2.45$ & $(5.79,0.01)$ & $\ldots$ & 20.68 & 20.50 & -14.76 & 20.28 & -14.98 \\
\hline
\end{tabular}

Note. Column 1: model components fit to the galaxy light-profile. Column 2: effective surface brightness of the spheroid, or central surface brightness of the disk or bar. Column 3: Effective half light radius. Column 4: disk or bar scale-length. Column 5: shape parameters of the Ferrers profile. Column 6: Sérsic index. Column 7: total observed, i.e., uncorrected, apparent magnitude ( $\mathrm{AB}$ mag). Column 8: Galactic-extinction and redshift-dimming corrected apparent magnitude. Column 9: absolute magnitude associated with column 8. Column 10: apparent magnitude from column 8 now further corrected for dust internal to the galaxy and the galaxy's inclination (Driver et al. 2008). Column 11: fully corrected absolute magnitude associated with column 10. The magnitudes correspond to the fitted components integrated to infinity.

Vaucouleurs et al. 1991) while the "SDSS Model" magnitude from Data Release 6 (Adelman-McCarthy et al. 2008) ${ }^{16}$ gave a $g^{\prime}$-band magnitude of $17.08 \mathrm{mag}$ (AB). Our preferred band is of course the redder $r^{\prime}$ band, however the $g^{\prime}$-band parameters derived here are used to a) check for consistency with the literature as just done and b) obtain a $g^{\prime}-r^{\prime}$ color for the bulge (see later in this section).

We explored the impact on the $r^{\prime}$-band bulge magnitude due to the uncertainty in the sky-background. Due to the large SDSS images, and thus the large number of $N$ sky pixels surrounding LEDA 87300, the uncertainty in the median sky value, equal to the standard deviation of the $N$ sky values $\left(\sigma_{\text {sky }}\right)$ divided by $\sqrt{N}$, is tiny. As such, it is the variation and uncertainty of the individual sky values in the pixels cooccupied by the galaxy which are important. Having $n$ pixels make up each isophote reduces this uncertainty to $\sigma_{\text {sky }} / n$ at each isophotal radius. Nonetheless, at large radii, this error in the sky-subtraction (such that the median sky-value for the isophote does not match the global median sky-value that was subtracted) dominates over the Poissonian scatter in the galaxy light. We repeated the galaxy decomposition with the uncertainty $\left(\sigma_{\text {sky }} / n\right)$ added to, and then subtracted from, the optimal light profile. Given that our fit was performed over the inner 10-16 arcsec, this did not have a big impact, with the spheroid's magnitude changing by $(-0.16,+0.12)$ mag. Adding and subtracting three times the uncertainty in the sky-background, the bulge magnitude changed by $(-0.37,+0.31) \mathrm{mag}$, the bar magnitude changed by just $(+0.05,-0.05) \mathrm{mag}$, and the disk magnitude changed by $(-0.24,+0.15)$ mag.

Unsurprisingly, changing the width of the PSF by a rather large $\pm 25 \%$ had almost no affect on the disk parameters, with the magnitude changing by just \pm 0.03 mag. While the bar was

${ }^{16}$ http://classic.sdss.org/dr6 found to be similarly stable, the spheroid magnitude changed by $\pm 0.4 \mathrm{mag}( \pm 45 \%)$ and the Sérsic index changed by $\pm 6 \%$.

We next corrected the observed, apparent magnitude for the usual influences. First, in the SDSS $g^{\prime}$ and $r^{\prime}$ bands there is 0.122 and $0.084 \mathrm{mag}$, respectively, of extinction in the direction of LEDA 87300 due to dust in our Galaxy (Schlafly \& Finkbeiner 2011). We also brightened the observed magnitudes by $5 \log (1+z)=0.06 \mathrm{mag}$, allowing for cosmological redshift dimming. Together, this gives us the first corrected magnitudes shown in Table 1 . These entries can be compared with the results from BRGG15. In particular, their stated "bulge" (barge) luminosity is 7.7 times brighter than our bulge luminosity, and more than 3 times as bright as our combined bulge plus bar luminosity. This is because of the way the bar in BRGG15's modeling resulted in a Sérsic component which extended beyond the end of the bar, eating into the disk light.

With the corrections mentioned above, we obtain a $g^{\prime}-r^{\prime}$ disk color of 0.35 , consistent with the value of $0.41 \pm 0.07$ reported in BRGG15. We additionally obtain a bulge color of $0.50 \pm 0.2$, and a blue bar color of $0.20 \pm 0.2$. Unlike the long bars of early-type spiral galaxies with bar colors that are similar to or redder than their host (e.g., Prieto et al. 2001), the shorter bars of late-type spiral galaxies tend to be bluer than their galaxy due to recent and ongoing star formation along the bar, revealing that they are young and growing (e.g., Elmegreen \& Elmegreen 1985; Kennicutt 1994; Phillips 1996; Gadotti \& de Souza 2006; Fanali et al. 2015 and references therein). The sites along the bar in LEDA 87300 with a $g^{\prime}-r^{\prime}$ color of $\sim 0.1-0.2$ (Figure 3 ) are indicative of this.

The second set of corrections that we apply are the combined inclination and dust corrections from Driver et al. (2008). At $R_{\text {maj }} \approx 16^{\prime \prime}$, beyond the radial range where the spiral arms dominate the ellipticity of LEDA 87300 , the ellipticity profile rapidly drops to about $0.1 \pm 0.1$ by $20^{\prime \prime}$, although the low ratio of signal to noise makes it hard to know the exact value. For a 
Table 2

Galaxy Model's Component Masses

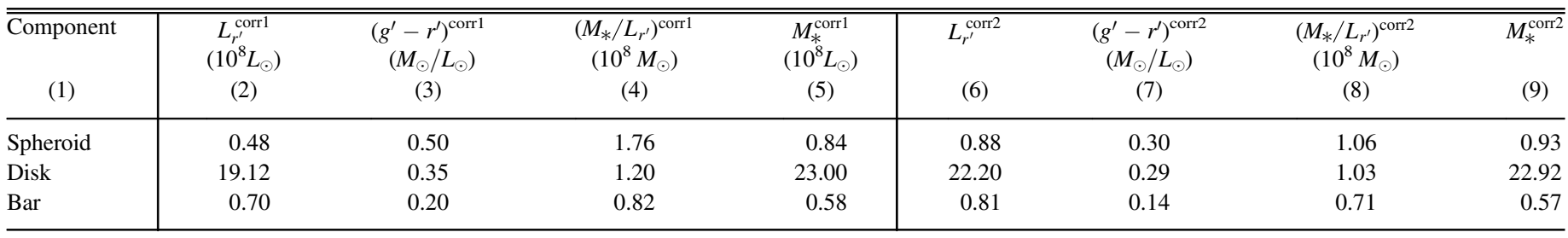

Note. Column 1: model components fit to the galaxy light profile. Column 2: $r^{\prime}$-band solar luminosity from column 8 of Table 1. Column 3: $g^{\prime}-r^{\prime}$ color derived from column 8 of Table 1. Column 4: adopted stellar mass-to-light ratio following Bell et al. (2003). Column 5: stellar mass. Column 6: $r^{\prime}$-band solar luminosity from column 10 of Table 1. Column 7: $g^{\prime}-r^{\prime}$ color derived from column 10 of Table 1. Column 8: adopted stellar mass-to-light ratio following Bell et al. (2003). Column 9: stellar mass.

disk inclination corresponding to an isophotal ellipticity of 0.1 (i.e., close to face-on), the bulge needs to be corrected/ brightened by 0.86 and $0.66 \mathrm{mag}$ in the $g^{\prime}$ and $r^{\prime}$ bands, while both the disk and bar (made out of the disk) should be brightened by 0.22 and $0.17 \mathrm{mag}$ in the $g^{\prime}$ and $r^{\prime}$ bands, respectively. ${ }^{17}$ The second last column in Table 1 shows the apparent magnitude of the galaxy's components after this additional correction is applied. These corrected apparent magnitudes were then converted into absolute magnitudes using a distance modulus of 35.26 , and used to obtain a second set of colors. The $r^{\prime}$-band absolute magnitudes were converted into solar luminosities using an absolute magnitude for the Sun of $M_{r^{\prime}, \odot}=4.65 \mathrm{AB}$ mag (Table 2).

\subsubsection{Stellar Masses}

We use the $g^{\prime}-r^{\prime}$ color to estimate the $r^{\prime}$-band stellar massto-light $\left(M_{*} / L_{r}\right)$ ratios. ${ }^{18}$ Following BRGG15, we use the prescription in Bell et al. (2003) which is such that

$$
\log _{10}\left(M_{*} / L_{r^{\prime}}\right)=1.097\left(g^{\prime}-r^{\prime}\right)-0.306,
$$

and reportedly has an accuracy of 20\% (Bell et al. 2003). Given that dust reddening roughly moves one along this relation (see Figure 6 in Bell et al. 2003, and Figure 13 in Driver et al. 2007), it can be applied to either the dust-reddened or the dust-corrected component's luminosity and color. The results are shown in Table 2, using both approaches, and the spheroid masses agree within $\sim 10 \%$. Our preference is to use the dust-corrected luminosities and masses (column 9 in Table 2). Using the stellar population synthesis model of Conroy et al. (2009), one has from Roediger \& Courteau (2015) that $\log \left(M_{*} / L_{r}\right)=1.497\left(g^{\prime}-r^{\prime}\right)-0.647$. This yields mass-to-light ratios for the spheroid which are some 30\%-40\% smaller than those obtained via the Bell et al. (2003) prescription. Given the uncertainties on the bulge luminosity arising from the uncertainty in (i) the sky-background, (ii) the PSF, (iii) the inclination of the disk, (iv) the $g^{\prime}-r^{\prime}$ color of the spheroid, (v) the uncertainty associated with the conversion from light-to-mass, and (vi) at some small level even the

\footnotetext{
17 If the adopted ellipticity is wrong by 0.1 , then the corrections to the bulge magnitude will change by just 0.02-0.06 mag; although it should be noted that while the mean corrective prescription from Driver et al. (2008) is appropriate for an ensemble of galaxies, its applicability for individual galaxies will vary. This makes it difficult to assess the error on the bulge magnitude due to dust. ${ }^{18} \mathrm{We}$ had hoped to use the $i^{\prime}$-band luminosity and the relation $\log \left(M_{*} / L_{i}\right)=-0.68+0.70\left(g^{\prime}-i^{\prime}\right)$ from Taylor et al. (2011), however, as noted before, the signal-to-noise ratio in the combined $i^{\prime}$-band image was too low.
}

adopted mask (Figure 4), we consider the spheroid stellar mass to be accurate to within a factor of 2 , although it could be as high as 3 if the inclination/dust correction was terribly wrong.

LEDA 87300 shows up in the GALaxy Evolution eXplorer All-Sky Survey Source Catalog (GALEXASC J152304.87 $+114552.0) \quad$ with $\quad$ NUV $\quad\left(\lambda_{\text {eff }}=0.227 \mu \mathrm{m}\right)=18.69 \pm$ 0.08 mag. Brightening for $\sim 0.20 \mathrm{mag}$ of Galactic extinction and $0.06 \mathrm{mag}$ of cosmological redshift dimming gives 18.43 mag. Using the galaxy $r^{\prime}$ magnitude from column 8 of Table 1, we have that NUV $-r^{\prime}=1.79$ for the galaxy. Together with the galaxy color $g^{\prime}-r^{\prime}=0.35$, LEDA 87300 resides in the middle of the "blue cloud" of star-forming galaxies (Chilingarian \& Zolotukhin 2012; their Figure 1), and is more blue than the average Sc galaxies (Chilingarian \& Zolotukhin 2012; their Figure 3), as expected for an Sm galaxy. Its ongoing star formation therefore supports the low stellar mass-to-light ratios of 0.71-1.76 that we find (Table 2).

For comparison with BRGG15, and therefore not considering the inclination and internal dust correction, we find that BRGG15 obtained a slightly fainter luminosity for their disk $\left(1.4 \times 10^{9} L_{\odot}\right)$ than us $\left(1.9 \times 10^{9} L_{\odot}:\right.$ Table 2 , column 2$)$, due to their fainter central surface brightness and smaller scalelength for their fitted disk (see their Figure 3). However, they also derived a redder disk and higher stellar mass-to-light ratio than us. This gave them a stellar mass of $10^{9.3+/-0.1} M_{\odot}$ $\left(=2.0_{-0.4}^{+0.5} \times 10^{9} M_{\odot}\right)$, which agrees well with our optimal disk mass of $2.3 \times 10^{9} M_{\odot}$. As noted before, BRGG15 obtained a notably $(7.7 \times)$ brighter "spheroid" $\left(3.7 \times 10^{8} L_{\odot}\right)$ than us $\left(0.48 \times 10^{8} L_{\odot}\right)$, due to their fitted model being biased by the bar light. BRGG15 report a "bulge" (barge) stellar mass of $10^{8.8 \pm 0.2} M_{\odot}\left(6.3_{-2.3}^{+3.7} \times 10^{8} M_{\odot}\right)$, while our optimal bulge mass is $0.84 \times 10^{8} M_{\odot}$, or 7.5 times less massive.

BRGG15 obtained a bulge-to-disk mass ratio $B / D=0.32$ $(B /$ Total $=0.24)$ which is what one typically finds in earlytype disk galaxies rather than in late-type spiral galaxies (e.g., Laurikainen et al. 2010). Our bulge-to-disk mass ratio is $\sim 4 \%$, in good agreement with values seen in Scd-Sm galaxies (Graham \& Worley 2008).

\section{ADDITIONAL PARAMETERS AND DATA}

\subsection{The Black Hole Mass}

Using a "reduced" virial factor $\epsilon=1$ (which equates to an $f$ factor equal to 4), BRGG15 reported black hole mass estimates ranging from (2.7-6.2) $\times 10^{4} f_{4} M_{\odot}$ when using three different techniques for modeling the narrow emission lines, plus an associated factor of 2.7 uncertainty arising from the empirical relations used to estimate virial black hole masses. This gives a 
black hole mass range from $10^{4} f_{4} M_{\odot}$ to $1.7 \times 10^{5} f_{4} \quad M_{\odot}$ about their adopted nominal value of $5 \times 10^{4} f_{4} \quad M_{\odot}$, where $f_{4}=f / 4$.

Building on Onken et al. (2004), who reported an $f$-factor of $5.5 \pm 1.7$, Graham et al. (2011) also investigated the optimal $f$ factor by calibrating the virial black hole masses obtained from reverberation mapping studies of AGNs against the $M_{\mathrm{bh}}-\sigma_{*}$ relation defined by other galaxies with directly measured black hole masses. Using both a "forward" and an "inverse" linear regression to construct the $M_{\mathrm{bh}}-\sigma_{*}$ relation-the latter is required for minimizing the sample selection bias that arises from the need to spatially resolve the black holes's sphere-ofinfluence-an $f$-factor of around 3 was derived for the full sample. Moreover, they reported why this was an upper limit because of several factors, including radiation pressure (Marconi et al. 2008, who also report $f \approx 3$ ). Repeating this analysis, the forward and inverse linear regressions from Park et al. (2012) yielded similar results to Graham et al. (2011), and an $f$-factor of 3 is commonly used in the literature (e.g., Kaspi et al. 2000; Jiang et al. 2011; Xiao et al. 2011). However when dealing only with AGNs in barred galaxies, Graham et al. (2011) found that the optimal $f$-factor ${ }^{19}$ was $2.3_{-0.6}^{+0.9}$ (and this may still be too high, see Shankar et al. 2016). The optimal virial black hole mass for LEDA 87300 is thus a factor of 2.3/ 4 times smaller than reported by BRGG15, and we therefore adopt a mass of $2.9 \times 10^{4} f_{2.3} M_{\odot}$ with a range from $(0.6-9.6) \times 10^{4} f_{2.3} M_{\odot}\left(\log \left[M_{\mathrm{bh}} f_{2.3}\right]=4.46_{-0.70}^{+0.52}\right)$. This is still consistent with the value adopted by BRGG15.

Our $M_{\mathrm{bh}} / M_{\mathrm{sph}, *}$ mass ratio for LEDA 87300 is $0.031 \%$, which is 2.4 times larger than the value of $0.013 \%$ obtained by BRGG15. Our ratio is 16 times smaller than the median value of $0.49 \%$ reported by Graham \& Scott (2013) for massive spheroids with partially depleted cores, and 22 times smaller than the median value of $0.68 \%$ reported by Savorgnan et al. (2016) for their early-type galaxy sample. ${ }^{20}$

\subsection{The Stellar Velocity Dispersion}

There is no available stellar velocity dispersion $\left(\sigma_{*}\right)$ for LEDA 87300. However BRGG15 measured a gas velocity dispersion of $27 \pm 5 \mathrm{~km} \mathrm{~s}^{-1}$ from the [N II] line. They assumed 0.15 dex of scatter and zero mean offset between measurements of the stellar and the gas velocity dispersion in other galaxies, and reported $\sigma_{*}=27_{-10}^{+12} \mathrm{~km} \mathrm{~s}^{-1}$. There is however a well known tendency for gas to have a lower velocity dispersion than stars (e.g., Vega Beltrán et al. 2001, their Figure 3; Barth et al. 2008, their Figure 10), with Ho (2009) reporting an average $\sigma_{[\mathrm{N} \mathrm{II}} / \sigma_{*}$ ratio of 0.8 . That is, the stellar velocity dispersion is typically $25 \%$ higher than the gas velocity dispersion. Although, this ratio is less clear for the Scd-Sm galaxies for which there is more scatter, with Figure 1 from Ho (2009) revealing that systems with $\sigma_{[\mathrm{N} \mathrm{II}} \approx 25-35 \mathrm{~km} \mathrm{~s}^{-1}$ have $\sigma_{*} \approx 23-100 \mathrm{~km} \mathrm{~s}^{-1}$. Figure $11 \mathrm{~d}$ from Xiao et al. (2011) reveals this same behavior at low velocity dispersions, and that one expects $\sigma_{*} \approx 30-50 \mathrm{~km} \mathrm{~s}^{-1}$ if $\sigma_{[\mathrm{N} \mathrm{I}]}=27 \mathrm{~km} \mathrm{~s}^{-1}$. We adopt this more constrained range rather than an average value of $50-60 \mathrm{~km} \mathrm{~s}^{-1}$ which might be expected from Ho (2009). Adding the $\pm 5 \mathrm{~km} \mathrm{~s}^{-1}$ measurement uncertainty

\footnotetext{
19 Graham et al. (2011) reported optimal $f$-factors of $5.4_{-1.2}^{+1.5}$ for unbarred galaxies, $2.3_{-0.6}^{+0.9}$ for barred galaxies (many of which may have pseudobulges), and $2.8_{-0.5}^{+0.7}$ for the ensemble.

20 The intrinsic scatter observed in these ratios is $\sim 0.4$ dex.
}
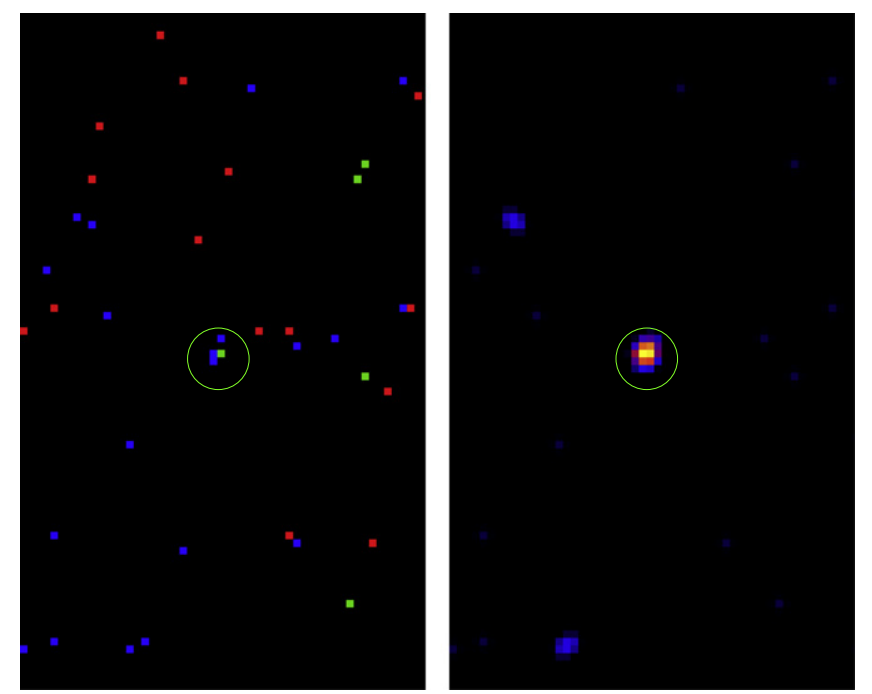

Figure 7. Left panel: Chandra X-ray Observatory ACIS-S photon image such that red $=0.3-1.0 \mathrm{keV}$, green $=1-2 \mathrm{keV}$ and blue $=2-7 \mathrm{keV}$. The green point within the green circle of radius equal to $2^{\prime \prime}$ centered on LEDA 87300 has an energy between 1.5 and $2 \mathrm{keV}$, while the three blue points within the circle have an energy between 2 and $5 \mathrm{keV}$. Right panel: the same image after smoothing with a Gaussian having $\sigma=3$ pixels. East is up and north is to the right.

in quadrature with a $\pm 10 \mathrm{~km} \mathrm{~s}^{-1}$ range, we conservatively adopt $\sigma_{*}=40 \pm 11 \mathrm{~km} \mathrm{~s}^{-1}$.

Given that spheroids with low Sérsic indices have rather flat, luminosity-weighted, velocity dispersion profiles (e.g., Graham $\&$ Colless 1997, their Figure 7), no discernible difference between the central $(R \approx 0)$ and effective $\left(R \leqslant R_{\mathrm{e}}\right)$ aperture velocity dispersion is expected for the spheroidal component of LEDA 87300. Of course the presence of disks and bars can bias attempts to measure the spheroid's velocity dispersion, and increasingly so as one moves to apertures of larger radii in disk galaxies. This is because one will increasingly acquire more of a galaxy velocity dispersion than a bulge velocity dispersion as the radial range is increased. For this reason, one should be wary of $\sigma_{R_{\mathrm{e}}}$ or $\sigma_{R_{\mathrm{e}} / 2}$, which may be systematically biased if the disk light and dynamics contribute more in galaxies with lower mass black holes.

\subsection{The X-Ray Data}

Given the current scarcity, and thus importance, of IMBHs, we have additionally re-analyzed the Chandra X-ray Observatory Advanced CCD Imaging Spectrometer (ACIS) data for LEDA 87300. We provide confirmation of the analysis in BRGG15, and therefore we do not discuss this data at any great length. Within the $5.5 \mathrm{hr}$ exposure, $4 \mathrm{X}$-ray photons were detected within a circle of radius $2^{\prime \prime}$ centered on the galaxy (see Figure 7). Given the average background count was just 0.25 for an area of this size, we have a $90 \%$ confidence limit of between 1 and 8 photons (Kraft et al. 1991). That is, we have at least a $90 \%$ detection of a point-like X-ray source in LEDA 87300. We acknowledge that "point-like" actually means within $\sim 1^{\prime \prime}$ or $\sim 500 \mathrm{pc}$, and thus the source of the $\mathrm{X}$-rays could be an AGN, a compact starburst nucleus, or a combination of both.

None of the four photons appeared in the $0.3-1.5 \mathrm{keV}$ band, they all had energies in the $1.5-5 \mathrm{keV}$ range, with just one having an energy between 1.5 and $2.0 \mathrm{keV}$. It is interesting that 


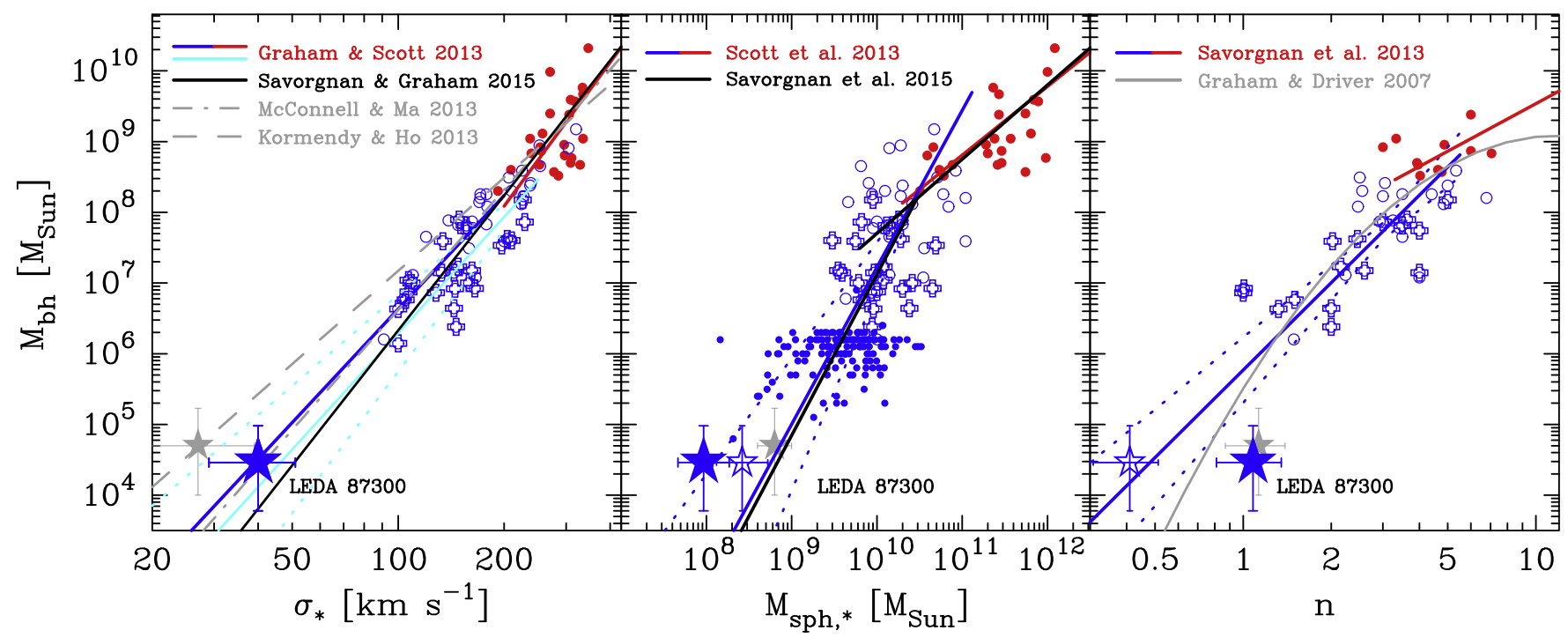

Figure 8. Left panel: $M_{\mathrm{bh}}-\sigma_{*}$ data and relations from Graham \& Scott (2013): core-Sérsic galaxies (red dots and red line); unbarred Sérsic galaxies (blue circles and blue line); barred galaxies (blue crosses and cyan line). The dotted cyan lines delineate the $1 \sigma$ uncertainty on the latter relation. The gray star shows the parameters for LEDA 87300 from BRGG15, while the blue star shows our parameters. Note: Kormendy \& Ho (2013) adjusted the velocity dispersions to create reduced velocity dispersion estimates within $R_{\mathrm{e}} / 2\left(\sigma_{*, R_{\mathrm{e}}} / 2\right)$ to which their $M_{\mathrm{bh}}-\sigma_{*}$ line pertains. Middle panel: $M_{\mathrm{bh}}-M_{\mathrm{sph}, *}$ diagram and relations from Scott et al. (2013): core-Sérsic galaxies (red); barred and unbarred Sérsic galaxies (blue). The AGNs shown here as small blue dots come from Graham \& Scott (2015) but were not used to derive the blue line. The dotted blue lines delineate the $1 \sigma$ uncertainty of the solid blue line. The gray star shows the "barge" (bar + bulge) parameters for LEDA 87300 from BRGG15, while the open blue star shows our parameters for the barge. The larger solid blue star shows our parameters for the bulge of LEDA 87300. Right panel: $M_{\mathrm{bh}}-n$ data and relations from Savorgnan et al. (2013).

there are no photons with energies in the $0.3-1.5 \mathrm{keV}$ band because it requires the soft band to be absorbed by a column density of neutral hydrogen of at least $5 \times 10^{21}$ and more likely $10^{22} \mathrm{~cm}^{-2}$. Assuming a plausible photon index ${ }^{21}$ for the slope of the X-ray spectrum somewhere in the range $\Gamma \approx 1.4-1.7$, one detected photon corresponds to a $2-10 \mathrm{keV}$ luminosity $L_{X} \approx 10^{39} \mathrm{erg} \mathrm{s}^{-1}$. As noted by BRGG15, the bolometric luminosity is therefore $\approx 4 \times 10^{40} \mathrm{erg} \mathrm{s}^{-1}$ if using $L_{\mathrm{bol}} / L_{2-10} \sim 10$ (Marconi et al. 2004; Vasudevan \& Fabian 2007).

The rest of the galaxy does not appear to contribute to the X-ray emission, and is not expected to. For a total galaxy stellar mass of $\sim 4 \times 10^{9} \quad M_{\odot}$, the expected X-ray luminosity from the low-mass $\mathrm{X}$-ray binaries (LMXBs) is $L_{X} \approx(8 \pm 1) \times 10^{28} M_{\text {galaxy }, *} \approx 3 \times 10^{38} \mathrm{erg} \mathrm{s}^{-1}$ (Gilfanov 2004). In the Chandra exposure, this would correspond to only $1 / 3$ of a photon in $5.5 \mathrm{hr}$. An additional component may come from high-mass X-ray binaries (HMXBs), however if the star formation rate is less than $\sim 0.5 M_{\odot} \mathrm{yr}^{-1}$, then we do not expect HMXBs to contribute significantly.

\section{DISCUSSION}

BRGG15 reported an ellipticity of 0.63 for their "bulge" component of LEDA 87300 and wrote that this value is in accord with expectations for pseudobulges. However because their "bulge" model was dominated by the bar (and perhaps the start of the spiral arms), they over-estimated the ellipticity of the actual spheroid which likely has an ellipticity half of this value (see Figure 6). As discussed in Graham (2014), pseudobulges are particularly difficult to identify, and as such we do not label the bulge component of LEDA 87300 one way or another.

\footnotetext{
${ }^{21}$ The photon index is defined as $d N / d E \propto E^{-\Gamma}$ where $N$ is the number flux of photons, e.g., Ishibashi \& Courvoisier (2010).
}

Figure 8 shows the location of LEDA 87300 in the $M_{\mathrm{bh}}-\sigma_{*}$, $M_{\mathrm{bh}}-M_{\mathrm{sph}, *}$, and $M_{\mathrm{bh}}-n$ diagrams. We have used the virial black hole mass estimated with the optimal virial factor for barred galaxies (Section 3.1), and we now discuss each panel in turn, starting with the $M_{\mathrm{bh}}-\sigma_{*}$ diagram.

BRGG15 showed that their black hole mass and adopted stellar velocity dispersion for LEDA 87300 placed it on the extrapolation of the $M_{\mathrm{bh}}-\sigma_{*}$ relation from Kormendy \& Ho (2013) which was constructed using a sample which Kormendy \& Ho (2013) considered not to be "pseudobulges." In the left panel of Figure 8, one can see that our slightly revised location of LEDA 87300 places it on the $M_{\mathrm{bh}}-\sigma_{*}$ relation from McConnell \& Ma (2013) for barred and unbarred galaxies combined. LEDA 87300 additionally agrees well with the $M_{\mathrm{bh}}-\sigma_{*}$ relations from Graham \& Scott (2013) for both barred and unbarred galaxies, which are themselves consistent with the relations presented in Graham et al. (2011).

Graham (2008) and $\mathrm{Hu}$ (2008) showed that barred galaxies -which may contain pseudobulges, classical bulges, or simultaneously both a classical bulge and a pseudobulge (e.g., Erwin et al. 2003)—have a tendency to sometimes be offset from the $M_{\mathrm{bh}}-\sigma_{*}$ relation in the direction of higher velocity dispersions, as explained by Hartmann et al. (2014). It was pointed out in Section 3.2 that the stellar velocity dispersion may be $\approx 50-60 \mathrm{~km} \mathrm{~s}^{-1}$. If correct, LEDA 87300 would remain within the $1 \sigma$ uncertainty of the $M_{\mathrm{bh}}-\sigma_{*}$ relation for barred galaxies shown in Figure 8. The uncertainties associated with the black hole mass and stellar velocity dispersion for LEDA 87300 , coupled with the uncertainty on the slope and intercept of the various $M_{\mathrm{bh}}-\sigma_{*}$ scaling relations, are such that we are not advocating any one relation over another, but expanding upon the consistency noted by BRGG15 between LEDA 87300 and the extrapolation of the $M_{\mathrm{bh}}-\sigma_{*}$ relations to lower velocity dispersions. Finally, we note that these relations may yet turn out to be upper envelopes 
(Batcheldor 2010), with Subramanian et al. (2016) presenting evidence for this with a sample of low surface brightness galaxies for which the black hole masses, estimated using the broad $\mathrm{H} \alpha$ line, fell well below the various $M_{\mathrm{bh}}-\sigma_{*}$ relations.

Including over 100 AGNs with virial black hole mass estimates from Jiang et al. (2011), Graham \& Scott (2015) extended the $M_{\mathrm{bh}}-M_{\mathrm{sph}, *}$ diagram from $M_{\mathrm{bh}}=10^{6} M_{\odot}$ down to $10^{5} M_{\odot}$. They revealed that the bulges of these AGNs follow the near-quadratic relation presented in Scott et al. (2013) for galaxies without partially depleted cores, i.e., for classical spheroids and pseudobulges alike. Mezcua et al. (2016) present additional AGNs supporting such a steep relation, bolstering support for theoretical models which predict a steep scaling relation at these masses (e.g., Fontanot et al. 2006, 2015; Dubois et al. 2012; Bellovary et al. 2014; Bonoli et al. 2014). Building on BRGG15, we extend the $M_{\mathrm{bh}}{ }^{-}$ $M_{\mathrm{sph}, *}$ diagram down to $M_{\mathrm{bh}} \sim 3 \times 10^{4} M_{\odot}$ through the inclusion of LEDA 87300 (Figure 8 , middle panel). The difference here is that we have used the (dust-corrected) bulge mass rather than a combination of the bulge plus bar mass. This had the effect of shifting LEDA 87300 to the left in this diagram. Although the previously reported location of LEDA 87300 (Figure 1) better matches the near-quadratic $M_{\mathrm{bh}} \propto M_{\mathrm{sph},{ }^{*}}^{2.22 \pm 0.58}$ relation from Scott et al. (2013), within the $1 \sigma$ uncertainties there remains agreement between LEDA 87300 and this steep relation. Finally, we note that while the use of the barge or galaxy luminosity will spread the data to the right in the $M_{\mathrm{bh}}-M_{\mathrm{sph}, *}$ diagram, star formation activity may also produce over-luminous bulges, as discussed by Busch et al. (2016).

LEDA 87300 can be seen to roughly follow the observed trend in the $M_{\mathrm{bh}}-n$ diagram (Figure 8, right panel). However LEDA 87300 is offset below the linear $M_{\mathrm{bh}}-n$ relation. For a Sérsic index $n_{\text {maj }}=1.08$, and a typical $25 \%$ uncertainty $^{22}$, the log-linear relation from Savorgnan et al. (2013) is such that $\log \left(M_{\mathrm{bh}} / M_{\odot}\right)=(7.73 \pm 0.12)+(4.11 \pm 0.72) \log (n / 3)$, and gives $\log \left(M_{\mathrm{bh}} / M_{\odot}\right)=5.91 \pm 0.64$, or equivalently $M_{\mathrm{bh}}=$ $8.1_{-6.2}^{+27.4} \times 10^{5} M_{\odot}$. The lower $1 \sigma$ bound to this mass range $\left(1.9 \times 10^{5} M_{\odot}\right)$ is two times higher than our upper range to the virial black hole mass $\left(0.96 \times 10^{5} f_{2.3} M_{\odot}\right)$. The curved $M_{\mathrm{bh}}-n$ relation from Graham \& Driver (2007) does better, but this is not our preferred solution because it does not separate galaxies into core-Sérsic versus Sérsic galaxies, or early-type versus late-type galaxies.

It should be born in mind that LEDA 87300 is just one data point, and the scaling relations are still being refined. Close agreements today may not be close agreements tomorrow, and thus one should be mindful of the uncertainty associated with the relations and the data. More IMBH data would be welcome, In this regard, while Reines et al. (2013) have identified candidate IMBHs in dwarf galaxies, and Sartori et al. (2015) are pursuing AGNs in low-mass galaxies, Graham \& Scott (2013) have identified targets in the small bulges of disk galaxies. Graham \& Scott (2013) named 41 candidate galaxies for hosting an IMBH based on the spheroid $K$-band magnitudes reported in the Dong \& De Robertis (2006) study of galaxies with low-luminosity AGNs. These spheroid magnitudes are being re-derived (B.C. Ciambur et al. 2016, in preparation) with new images having better spatial resolution than the 2 "! 5

\footnotetext{
${ }^{22}$ Equation (3) from Graham and Driver et al. (2007) reveals how this uncertainty propagates through to the predicted black hole mass. An intrinsic scatter of 0.3 dex in the $\log \left(M_{\mathrm{bh}}\right)$ direction has been used here.
}

Two Micron All Sky Survey (2MASS) data ${ }^{23}$ images used by Dong \& De Robertis (2006). This will provide improved spheroid magnitudes and thus better black hole mass estimates. An observational campaign (N.A. Webb et al. 2016, in preparation) to collect $5 \mathrm{GHz}$ radio data and X-ray data will provide an independent means for estimating/confirming these black hole masses via the "fundamental plane of black hole activity" (Merloni et al. 2003; Falcke et al. 2004; Dong \& Wu 2015; Liu et al. 2016; Nisbet \& Best 2016) and hopefully enable the further population of the black hole scaling diagrams at masses below $10^{5} M_{\odot}$.

This research was supported under the Australian Research Councils funding scheme (FT110100263). This research has made use of the NASA/IPAC Extragalactic Database (NED). Funding for SDSS-III has been provided by the Alfred P. Sloan Foundation, the Participating Institutions, the National Science Foundation, and the U.S. Department of Energy Office of Science.

\section{REFERENCES}

Adelman-McCarthy, J. K., Agüeros, M. A., Allam, S. S., et al. 2008, ApJS, 175,297

Ahn, C. P., Alexandroff, R., Allende Prieto, C., et al. 2012, ApJS, 203, 21

Alexander, T., \& Natarajan, P. 2014, Sci, 345, 1330

Baldassare, V. F., Reines, A. E., Gallo, E., \& Greene, J. E. 2015, ApJL, 809, L14 (BRGG15)

Barth, A. J., Greene, J. E., \& Ho, L. C. 2008, AJ, 136, 1179

Batcheldor, D. 2010, ApJL, 711, L108

Beifiori, A., Courteau, S., Corsini, E. M., \& Zhu, Y. 2012, MNRAS, 419, 2497

Belczynski, K., Bulik, T., Fryer, C. L., et al. 2010, ApJ, 714, 1217

Bell, E. F., McIntosh, D. H., Katz, N., \& Weinberg, M. D. 2003, ApJS, 149, 289

Bellovary, J., Holley-Bockelmann, K., Gültekin, K., et al. 2014, MNRAS, 445, 2667

Bonoli, S., Mayer, L., \& Callegari, S. 2014, MNRAS, 437, 1576

Busch, G., Fazeli, N., Eckart, A., et al. 2016, A\&A, in press (arXiv:1511.00904)

Caon, N., Capaccioli, M., \& D’Onofrio, M. 1993, MNRAS, 265, 1013

Chilingarian, I. V., \& Zolotukhin, I. Y. 2012, MNRAS, 419, 1727

Ciambur, B. C. 2015, ApJ, 810, 120

Colbert, E. J. M., \& Mushotzky, R. F. 1999, ApJ, 519, 89

Combes, F., Debbasch, F., Friedli, D., \& Pfenniger, D. 1990, A\&A, 233, 82

Combes, F., \& Sanders, R. H. 1981, A\&A, 96, 164

Conroy, C., Gunn, J. E., \& White, M. 2009, ApJ, 699, 486

Cseh, D., Webb, N. A., Godet, O., et al. 2015, MNRAS, 446, 3268

Damjanov, I., McCarthy, P. J., Abraham, R. G., et al. 2009, ApJ, 695, 101

Davis, S. W., Narayan, R., Zhu, Y., et al. 2011, ApJ, 734, 111

de Vaucouleurs, G., de Vaucouleurs, A., Corwin, H. G., Jr., et al. 1991, Third Reference Catalogue of Bright Galaxies (New York: Springer)

Dong, A.-J., \& Wu, Q. 2015, MNRAS, 453, 3447

Dong, X. Y., \& De Robertis, M. M. 2006, AJ, 131, 1236

Drinkwater, M. J., Gregg, M. D., Hilker, M., et al. 2003, Natur, 423, 519

Driver, S. P., Popescu, C. C., Tuffs, R. J., et al. 2007, MNRAS, 379, 1022

Driver, S. P., Popescu, C. C., Tuffs, R. J., et al. 2008, ApJL, 678, L101

Dubois, Y., Devriendt, J., Slyz, A., \& Teyssier, R. 2012, MNRAS, 420, 2662

Ekşi, K. Y., Andaç, İ. C., Çıkıntoğlu, S., et al. 2015, MNRAS, 448, L40

Elmegreen, B. G., \& Elmegreen, D. M. 1985, ApJ, 288, 438

Erwin, P., Beltrán, J. C. V., Graham, A. W., \& Beckman, J. E. 2003, ApJ, 597, 929

Fabrika, S., Ueda, Y., Vinokurov, A., Sholukhova, O., \& Shidatsu, M. 2015, NatPh, 11, 551

Falcke, H., Körding, E., \& Markoff, S. 2004, A\&A, 414, 895

Fanali, R., Dotti, M., Fiacconi, D., \& Haardt, F. 2015, MNRAS, 454, 3641

Farrell, S. A., Servillat, M., Gladstone, J. C., et al. 2014, MNRAS, 437, 1208

Farrell, S. A., Webb, N. A., Barret, D., Godet, O., \& Rodrigues, J. M. 2009, Natur, 460, 73

Feng, H., \& Soria, R. 2011, Natur, 55, 166

23 http://www.ipac.caltech.edu/2mass 
Ferrarese, L., \& Merritt, D. 2000, ApJL, 539, L9

Ferrers, N. M. 1877, QJPAM, 14, 1

Fontanot, F., Monaco, P., Cristiani, S., \& Tozzi, P. 2006, MNRAS, 373, 1173

Fontanot, F., Monaco, P., \& Shankar, F. 2015, MNRAS, 453, 4112

Freeman, K. C. 1970, ApJ, 160, 811

Gadotti, D. A., \& de Souza, R. E. 2006, ApJS, 163, 270

Gao, Y., Wang, Q. D., Appleton, P. N., \& Lucas, R. A. 2003, ApJL, 596, L171

Garnier, R., Paturel, G., Petit, C., Marthinet, M. C., \& Rousseau, J. 1996, A\&AS, 117, 467

Gebhardt, K., Bender, R., Bower, G., et al. 2000, ApJL, 539, L13

Gilfanov, M. 2004, MNRAS, 349, 146

Graham, A. W. 2008, ApJ, 680, 143

Graham, A. W. 2012, ApJ, 746, 113

Graham, A. W. 2014, in ASP Conf. Ser. 480, Structure and Dynamics of Disk Galaxies, ed. M. S. Seigar, \& P. Treuthardt (San Francisco, CA: ASP), 185

Graham, A. W. 2015, in Galactic Bulges, Vol. 418, ed. E. Laurikainen, R. F. Peletier, \& D. Gadotti (Berlin: Springer)

Graham, A. W. 2016, in IAU Symp. 312, Star Clusters and Black Holes in Galaxies Across Cosmic Time, ed. R. Spurzem et al. in press (arXiv:1412.5715)

Graham, A. W., \& Colless, M. 1997, MNRAS, 287, 221

Graham, A. W., \& Driver, S. P. 2005, PASA, 22, 118

Graham, A. W., \& Driver, S. P. 2007, ApJ, 655, 77

Graham, A. W., Dullo, B. T., \& Savorgnan, G. A. D. 2015, ApJ, 804, 32

Graham, A. W., Erwin, P., Caon, N., \& Trujillo, I. 2001, ApJL, 563, L11

Graham, A. W., Onken, C. A., Athanassoula, E., \& Combes, F. 2011, MNRAS, 412, 2211

Graham, A. W., \& Scott, N. 2013, ApJ, 764, 151

Graham, A. W., \& Scott, N. 2015, ApJ, 798, 54

Graham, A. W., \& Worley, C. C. 2008, MNRAS, 388, 1708

Greene, J. E., \& Ho, L. C. 2004, ApJ, 610, 722

Greene, J. E., \& Ho, L. C. 2005, ApJ, 630, 122

Gualandris, A., \& Merritt, D. 2008, ApJ, 678, 780

Häring, N., \& Rix, H.-W. 2004, ApJL, 604, L89

Hartmann, M., Debattista, V. P., Cole, D. R., et al. 2014, MNRAS, 441, 1243

Haynes, M. P., Giovanelli, R., Martin, A. M., et al. 2011, AJ, 142, 170

Ho, L. C. 2009, ApJ, 699, 638

Hohl, F. 1975, in IAU Symp., 69, Dynamics of Stellar Systems, ed. A. Hayli (Dordrecht: Reidel), 349

Hu, J. 2008, MNRAS, 386, 2242

Ishibashi, W., \& Courvoisier, T. J.-L. 2010, A\&A, 512, A58

Jedrzejewski, R. I. 1987, MNRAS, 226, 747

Jiang, Y.-F., Greene, J. E., Ho, L. C., Xiao, T., \& Barth, A. J. 2011, ApJ, 742,68

Johnson, J. L., Whalen, D. J., Li, H., \& Holz, D. E. 2013, ApJ, 771, 116

Kaaret, P., \& Feng, H. 2013, ApJ, 770, 20

Kaspi, S., Smith, P. S., Netzer, H., et al. 2000, ApJ, 533, 631

Kennicutt, R. C., Jr. 1994, in Mass-Transfer Induced Activity in Galaxies, ed. I. Shlosman (Cambridge: Cambridge Univ. Press), 131

Komossa, S., \& Merritt, D. 2008, ApJL, 683, L21

Kormendy, J., \& Ho, L. C. 2013, ARA\&A, 51, 511

Kraft, R. P., Burrows, D. N., \& Nousek, J. A. 1991, ApJ, 374, 344

Lanzoni, B. 2015, in IAU Symp. 312, Star Clusters and Black Holes in Galaxies Across Cosmic Time, ed. R. Spurzem et al. (Cambridge: Cambridge Univ. Press), arXiv:1502.04479

Laor, A. 1998, ApJL, 505, L83

Laor, A. 2001, ApJ, 553, 677

Läsker, R., Ferrarese, L., \& van de Ven, G. 2014a, ApJ, 780, 69

Läsker, R., Ferrarese, L., van de Ven, G., \& Shankar, F. 2014b, ApJ, 780, 70

Lasota, J.-P., King, A. R., \& Dubus, G. 2015, ApJL, 801, L4

Latif, M. A., Schleicher, D. R. G., Schmidt, W., \& Niemeyer, J. 2013, MNRAS, 433, 1607

Laurikainen, E., Salo, H., Buta, R., Knapen, J. H., \& Comerón, S. 2010, MNRAS, 405, 1089

Liu, J., Orosz, J., \& Bregman, J. N. 2012, ApJ, 745, 89

Liu, J.-F., Bregman, J. N., Bai, Y., Justham, S., \& Crowther, P. 2013, Natur, 503,500

Liu, X., Han, Z., \& Zhang, Z. 2016, Ap\&SS, 361, 9

Lupi, A., Haardt, F., Dotti, M., et al. 2016, MNRAS, 456, 2993

Lützgendorf, N., Kissler-Patig, M., Neumayer, N., et al. 2013, A\&A, 555, A26

Madau, P., Haardt, F., \& Dotti, M. 2014, ApJL, 784, L38

Madau, P., \& Rees, M. J. 2001, ApJL, 551, L27

Magorrian, J., Tremaine, S., Richstone, D., et al. 1998, AJ, 115, 2285

Marconi, A., Axon, D. J., Maiolino, R., et al. 2008, ApJ, 678, 693
Marconi, A., \& Hunt, L. K. 2003, ApJL, 589, L21

Marconi, A., Risaliti, G., Gilli, R., et al. 2004, MNRAS, 351, 169

Marquardt, D. 1963, SIAM, 11, 431

McConnell, N. J., \& Ma, C.-P. 2013, ApJ, 764, 184

McLure, R. J., \& Dunlop, J. S. 2002, MNRAS, 331, 795

Merloni, A., Heinz, S., \& di Matteo, T. 2003, MNRAS, 345, 1057

Merritt, D., Schnittman, J. D., \& Komossa, S. 2009, ApJ, 699, 1690

Mezcua, M., Civano, F., Fabbiano, G., Miyaji, T., \& Marchesi, S. 2016, ApJ, 817, 20

Mezcua, M., Roberts, T. P., Lobanov, A. P., \& Sutton, A. D. 2015, MNRAS, 448, 1893

Miller, J. M., Walton, D. J., King, A. L., et al. 2013, ApJL, 776, L36

Miller, M. C., \& Hamilton, D. P. 2002, MNRAS, 330, 232

Mould, J. R., Huchra, J. P., Freedman, W. L., et al. 2000, ApJ, 529, 786

Nisbet, D. M., \& Best, P. N. 2016, MNRAS, 455, 2551

Oka, T., Mizuno, R., Miura, K., \& Takekawa, S. 2016, ApJL, 816, L7

Onken, C. A., Ferrarese, L., Merritt, D., et al. 2004, ApJ, 615, 645

Park, D., Kelly, B. C., Woo, J.-H., \& Treu, T. 2012, ApJS, 203, 6

Pasham, D. R., Cenko, S. B., Zoghbi, A., et al. 2015, ApJL, 811, L11

Pasham, D. R., Strohmayer, T. E., \& Mushotzky, R. F. 2014, Natur, 513, 74

Paturel, G., Fouque, P., Bottinelli, L., \& Gouguenheim, L. 1989, A\&AS, 80, 299

Paturel, G., Petit, C., Garnier, R., \& Prugniel, P. 2000, A\&AS, 144, 475

Peng, C. Y., Ho, L. C., Impey, C. D., \& Rix, H.-W. 2010, AJ, 139, 2097

Phillips, A. C. 1996, in ASP Conf. Series, Vol. 91, Barred Galaxies, ed. R. Buta et al. (San Francisco, CA: ASP), 44

Pohlen, M., Dettmar, R.-J., Lütticke, R., \& Aronica, G. 2002, A\&A, 392, 807

Pohlen, M., \& Trujillo, I. 2006, A\&A, 454, 759

Portegies Zwart, S. F., \& McMillan, S. L. W. 2002, ApJ, 576, 899

Prieto, M., Aguerri, J. A. L., Varela, A. M., \& Muñoz-Tuñón, C. 2001, A\&A, 367, 405

Reines, A. E., Greene, J. E., \& Geha, M. 2013, ApJ, 775, 116

Roediger, J. C., \& Courteau, S. 2015, MNRAS, 452, 3209

Sabra, B. M., Saliba, C., Abi Akl, M., \& Chahine, G. 2015, ApJ, 803, 5

Sani, E., Marconi, A., Hunt, L. K., \& Risaliti, G. 2011, MNRAS, 413, 1479

Sartori, L. F., Schawinski, K., Treister, E., et al. 2015, MNRAS, 454, 3722

Savorgnan, G. A. D., \& Graham, A. W. 2015, MNRAS, 446, 2330

Savorgnan, G. A. D., \& Graham, A. W. 2016a, ApJS, 222, 10

Savorgnan, G. A. D., \& Graham, A. W. 2016b, MNRAS, 457, 320

Savorgnan, G. A. D., Graham, A. W., Marconi, A., et al. 2013, MNRAS, 434,387

Savorgnan, G. A. D., Graham, A. W., Marconi, A., \& Sani, E. 2015, ApJ, in press (arXiv:1511.07437)

Schlafly, E. F., \& Finkbeiner, D. P. 2011, ApJ, 737, 103

Schombert, J. M., Bothun, G. D., Schneider, S. E., \& McGaugh, S. S. 1992, AJ, 103, 1107

Scott, N., Graham, A. W., \& Schombert, J. 2013, ApJ, 768, 76

Secrest, N. J., Satyapal, S., Gliozzi, M., et al. 2012, ApJ, 753, 38

Sérsic, J.-L. 1963, BAAA, 6, 41

Seth, A., van den Bosch, R., Mieske, S., et al. 2014, Natur, 513, 398

Shankar, F., Mariangela, B., Sheth, R. K., et al. 2016, MNRAS, submitted

Shuder, J. M. 1981, ApJ, 244, 12

Soria, R., Hau, G. K. T., Graham, A. W., et al. 2010, MNRAS, 405, 870

Subramanian, S., Ramya, S., Das, M., et al. 2016, MNRAS, 455, 3148

Sutton, A. D., Roberts, T. P., Walton, D. J., Gladstone, J. C., \& Scott, A. E 2012, MNRAS, 423, 1154

Taylor, E. N., Hopkins, A. M., Baldry, I. K., et al. 2011, MNRAS, 418, 1587

Tremaine, S., Gebhardt, K., Bender, R., et al. 2002, ApJ, 574, 740

Tully, R. B. 1988, Nearby Galaxies Catalog (Cambridge: Cambridge Univ. Press)

van den Bosch, R. C. E., Gebhardt, K., Gültekin, K., et al. 2012, Natur, 491, 729

Vasudevan, R. V., \& Fabian, A. C. 2007, MNRAS, 381, 1235

Vega Beltrán, J. C., Pizzella, A., Corsini, E. M., et al. 2001, A\&A, 374, 394

Vika, M., Driver, S. P., Cameron, E., Kelvin, L., \& Robotham, A. 2012 MNRAS, 419, 2264

Webb, N., Cseh, D., Lenc, E., et al. 2012, Sci, 337, 554

Webb, N. A., Barret, D., Godet, O., et al. 2010, ApJL, 712, L107

Webb, N. A., Godet, O., Wiersema, K., et al. 2014, ApJL, 780, L9

Wright, E. L. 2006, PASP, 118, 1711

Wyller, A. A. 1970, ApJ, 160, 443

Xiao, T., Barth, A. J., Greene, J. E., et al. 2011, ApJ, 739, 28

Zhou, X.-L. 2015, NewA, 37, 1 